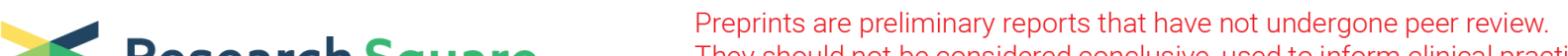 Research Square They should not be considered conclusive, used to inform clinical practice, or referenced by the media as validated information.
}

\section{Exogenous microRNA 322-5p Reduced Neuronal Inflammation via the TRAF6/IRF5/NF-kB Axis and Restored GAD1/GABA Expression in a Pilocarpine- induced Epileptic Rat Model}

Qin Zhou

Zhejiang Provincial People's Hospital https://orcid.org/0000-0003-4965-4614

Baomei He

Zhejiang Provincial People's Hospital

Haibo Kong

Zhejiang Provincial People's Hospital

Yanru Wang

Zhejiang Provincial People's Hospital

Wenlan Wang ( $\square$ wangwenlan@hmc.edu.cn )

Zhejiang Provincial People's Hospital

\section{Research}

Keywords: Epilepsy, miR-322-5p//TRAF6/IRF1/NF-kB signaling, inflammation, therapeutics development

Posted Date: July 15th, 2021

DOl: https://doi.org/10.21203/rs.3.rs-711777/v1

License: (c) (1) This work is licensed under a Creative Commons Attribution 4.0 International License.

Read Full License 


\section{Abstract}

Objective: MicroRNAs (miRNAs) are small noncoding RNAs that control gene expression at the posttranscriptional level. Some dysregulated miRNAs have been shown to play essential roles in epileptic development. This study aimed to determine if microRNA-322-5p regulates seizure and seizure damage by targeting the NFKB-TLR4 associated inflammatory signaling pathway.

Methods: Pilocarpine-induced epileptic rat model was established. Immunohistochemical staining demonstrated the pathology of epilepsy. The expression of microRNA-322-5p, inflammatory markers, NFkB, TLR4, IL-1 $\beta$ and IL-6, and synaptic inhibitory molecules, GAD1 and GABA, were assessed by a quantitative polymerase chain reaction, and western blotting, respectively.

Results: The expression of microRNA-322-5p was significantly decreased in the SE (status epilepticus) rats compared with the normal counterparts. The reduction of miR-322-5p was accompanied by an increased level of pro-inflammatory cytokines such as IL- 6 and TNF- $\alpha$ via increased NF-kB expression and reduced GAD1 and GABA expression. The exogenously increased miR-322-5p level by mimic molecules significantly reduced the inflammatory profiles and increased GAD1 and GABA expressions in the S.E. rat brain compared to nontreated counterparts.

Conclusions: Our findings suggest that the restoration of miR-322-5p resulted in a significantly reduced TRL4/IRF1/NF-kb associated inflammatory circuit and increased GAD1 and GABA expression. These findings suggest that miR-322-5p induction may be of therapeutic potential for neural damage as a result of repeated epileptic episodes.

\section{Background}

Status epilepticus (SE) is a common acute and critical illness in children. If it is not treated in time, it can cause irreversible brain damage due to high fever, circulatory failure, or neuronal excitotoxic damage. Long-term loss of learning and memory, mental retardation, mental retardation, and paralysis will remain. In severe cases, it can endanger the lives of children, with high mortality and disability rates. According to the survey, the annual incidence of SE in all age groups is (10-40)/100,000 (1). SE is seen in $10 \%-20 \%$ of children with epilepsy, and the tonic-clonic status is the most common. The longer the duration of SE attack, the more serious the brain damage caused, and the fatality rate of SE is close to $20 \%$ (1). Therefore, active, rapid, and effective control of epileptic seizures is the key to saving patients' lives and improving their prognosis, especially in the field of pediatrics.

Emerging evidence indicates that dysregulation of inflammatory circuits in the lesion sites represents a critical factor in the development of epilepsy. However, it remains underappreciated as to how the unbalanced regulation of inflammation contributes to the development of epilepsy and subsequent drug resistance (2). A strong association between inflammatory circuits and decreased activity of $\mathrm{Y}^{-}$ Aminobutyric acid (GABA), a major inhibitory neurotransmitter, has been shown in epileptogenesis $(3,4)$ Thus, GABA receptors and/or associated signaling have been the targets for therapeutic development. In 
addition, the level of GABA is regulated by a key enzyme, glutamate decarboxylase 1 (GAD1). Together, these signaling pathways and enzymes play a significant role in the development of epileptic activities (3). The etiology of epilepsy remains complex, the neuroinflammatory responses could arise from a variety of different sources, such as head trauma, infections of the central nervous system (CNS), CNS cancer and the aging process all can contribute to the development of epilepsy. The activation of Toll-like receptors (TLRs) via a nuclear protein released from dying cells such as high-mobility group box-1 (HMGB1) has been shown to contribute to chronic epilepsy in rat models as well as in human epileptic tissues. For instance, pilocarpine, a chemoconvulsant that induces epilepsy with chronic seizures, was mediated by the amplification of TRL4 associated signaling. However, the regulation of TRL4 signaling in the context of epilepsy has not been fully appreciated.

MicroRNAs (or miRs) represent a group of small noncoding RNAs that can specifically bind to the $3^{\prime}$ untranslated region (3'UTR) of mRNA targets and subsequently silence the expression by way of inhibiting the translation of the target proteins(5). Emerging evidence indicates that miRs also contribute to the development of epileptogenesis. For instance, a previous study reported specific subsets of miRNAs that were upregulated and downregulated in a status epilepticus (S.E.) rat model (6). In our search of public databases, we identified miR-322-5p as one of the most decreased microRNA species in the S.E. rat brain. However, its role in epilepsy remains mostly unexplored. Connecting the importance of neuroinflammation, the role of GABA and its related signaling pathways in epilepsy, in this study, we aimed to demonstrate the relationship between miR-322-5p and its targeted signaling circuits. Finally, we investigated the feasibility of translating our research results to future clinical applications.

\section{Materials And Methods}

\section{Pilocarpine induced epileptic rat model}

Sprague-Dawley rats (male, 20-day of age) were purchased from SLAC (Shanghai, People's Republic of China) and maintained in the following conditions: 12-hour light/dark cycle at a constant temperature of $23^{\circ} \mathrm{C} \pm 2^{\circ} \mathrm{C}$ and with food and water ad libitum. All procedures were conducted according to the regulations of Animal experiment facility and approved by the Experimental Animal Ethical Committee of Zhejiang Provincial People's Hospital. Briefly, 48 rats were used to produce an epilepsy model through lithium chloride ( $\mathrm{LiCl}$ injection ( $3 \mathrm{mEq} / \mathrm{kg}$, i.p.) followed by an injection of $1 \mathrm{mg} / \mathrm{kg}$ methyl scopolamine bromide (18 hours later). 30 minutes after methyl scopolamine bromide injection, rats were injected with pilocarpine hydrochloride (100 mg/kg, i.p.) (7). Rats were then monitored to detect seizure activity for $2 \mathrm{~h}$ following pilocarpine administration. Seizures were determined and recorded in five stages based on Racine's scale with slight modifications. In short, stage 1: face and vibrissae twitching, ear rubbing on forepaws, chewing. Stage 2: nodding of the head, unilateral limb clonus. Stage 3: limb clonus, mild convulsions. Stage 4: rearing with bilateral forelimb clonus, tail hypertension, lockjaw, and whole-body convulsions. Finally, stage 5: rearing with body convulsions and collapsing with body rigidity. The onset of S.E. was defined when stages 4 or 5 were reached, and the development of continuous motor 
activity(8). Chloral hydrate ( $400 \mathrm{mg} / \mathrm{kg}$, i.p) and atropine ( $1 \mathrm{mg} / \mathrm{kg}$, i.p) was subsequently injected 30 minutes post onset of S.E. to attenuate seizure activity (9).

\section{Animal grouping and intervention}

After successful modeling of SE rats, the rats were divided into two experiments: Experiment 1, the expression of miR-322-5p, TRL4, TRAF6, RelA (NF-kb subunit) and IRF1 in SE rats at different times; SE rats divided into 4 subgroups of 1 week, 2 weeks, 3 weeks and 4 weeks ( $n=8$ in each group). The control group consisted of eight normal rats. Experiment two, the effect of overexpression of miR-322-5p. The SE rats were divided into SE + miR-322-5p intervention group and SE + control(scrambled) ( $n=8$ in each group). Overexpression of miR-322-5p, which targets TRAF6 and IRF5 mRNAs were designed and synthesized by Shanghai GeneChem Co., Ltd. (Shanghai, China). Please refer to Table 1 for the primer sequences used in this study. The microinjection of exogenous miR-322-5p was performed according to a previously established protocol. In short, rats were microinjected with $10 \mu \mathrm{lmiR}-322-5 \mathrm{p}(50 \mu \mathrm{mol} / \mathrm{I})$ via a pre-implanted cannula, 30 minutes after SE modelling for 3 consecutive days. Following each injection, the needle was left in place for 5 min to allow complete diffusion of the injected material. The SE+control (scrambled) group received $10 \mu \mathrm{l} \mathrm{scrambled} \mathrm{RNA}(50 \mu \mathrm{mol} / \mathrm{l})$ and served as the negative control. The control group and the epilepsy group received the same volume of normal saline for microinjection. One week after treatment, all animals were put to death and specimens were kept.

\section{Quantitative PCR reactions}

Neuoinflammatory genes and miR-322-5p expression profiles were measured by real-time PCR reactions. Total RNAs were extracted using a Trizol kit and the quality was checked by OD260/OD280 ratio (RNA samples with the $260 / 280$ ratio $>1.8$ were used for experiments). cDNA was then reversed and transcribed and the qPCR reactions were performed using a LightCycler 96 (Hoffman-La Roche Ltd., Basel, Switzerland) following the preset procedure: $95^{\circ} \mathrm{C}$ initial denaturation for 5 minutes, $95^{\circ} \mathrm{C}$ denaturation for 30 seconds, $58^{\circ} \mathrm{C}$ annealing for 30 seconds, and $72^{\circ} \mathrm{C}$ extension 30 seconds for 30 cycles. The expressions of the genes of interest were determined by the $-\Delta \Delta C T$ method. All primer sequences can be found in Table 1.

\section{Immunohistochemistry}

Rats were first anaesthetized by $10 \%$ chloral hydrate (i.p. injection) and perfused with cold $4 \%$ paraformaldehyde (Sigma, China) through the auricula sinistra for 30 min. Rats were then sacrificed by decapitation, and the hippocampal tissues were collected and fixed in $4 \%$ paraformaldehyde for $2 \mathrm{~h}$. Subsequently, the tissues were paraffin-embedded, sliced ( $6 \mu \mathrm{m}$-thick), and stained. Nissl staining procedures were performed according to a previously established method(10).

\section{Western blotting experiments}

Total cellular proteins from the brain homogenates were extracted using RIPA buffer (Santa Cruz Biotechnology, CA, USA) supplemented with a cocktail of protease inhibitors (Roche, Basel, Switzerland). 
The concentration of the protein extracts was quantified by a BCA Protein Assay Kit (Pierce, Rockford, IL, USA). Each sample $(40 \mu \mathrm{g})$ was loaded into a $10 \%$ sodium dodecyl sulfate-polyacrylamide gel (SDSPAGE), electrophoresed, and transferred to a polyvinylidene fluoride (PVDF) membrane (Millipore, Bedford, MA, USA). PVDF membranes were then first blocked with $5 \%$ BSA ( $1 \mathrm{~h}$, room temperature) and incubated with primary antibodies in the cold overnight. The primary antibodies: anti-TRL4 (\#14358), anti-TRAF6 (\#8028), anti-RelA (\#3033), anti-IRF5 (\#20261), anti-IL-1ß (\#31202), anti-IL-6 (\#3), anti-GABA (\#8921), anti-GAD1 (\#63080), anti-GAPDH (\#4970) were all purchased from Cell Signaling Technology (Beverly, MA, USA). The PVDF membranes were subsequently probed with HRP-conjugated secondary antibodies (Sigma-Aldrich) at a 1: 6000 dilution (1 h, room temperature). Finally, an ECL kit (Beyotime Biotechnology, USA) was used to detect the protein-antibody signals.

\section{Results}

\section{Status epilepticus (S.E.) rat brain showed an elevated TRL4/NF-kB expression profile and decreased GABA expression.}

A pilocarpine-induced epileptic rat model was established to examine the role of TRL4/NF-kB circuit in epileptogenic development. The representative hippocampal section from the S.E. rat brain showed disorganized neurons compared to their control counterparts (Figure 1A). The amount of GABA was significantly reduced in the S.E. rat homogenates for the validation of the model (insert, Figure 1B). We then examined the components of the Toll-like receptor 4 (TRL4) inflammatory circuit and found that there was a time-dependent increase in the expression of TRL4, TRAF6, RelA (NF-kb subunit) and IRF1 (Figure 1C), suggesting the chronic inflammation had occurred in the S.E. brains.

\section{A negative association was observed between TRL4/TRAF6 circuit and the level of miR-322-5p in S.E. rat brain tissue}

Subsequently, we searched a public database of microRNA profilings in S.E. rats (11)and found that one of the most affected microRNA speices was miR-322-5p (Figure 2A). Based on three target gene prediction tools (miRmap, TargetScan, and PITA), we identified that one of the key TLR4/NF-kB signaling components, TRAF6, was the target of miR-322-5p where miR-322-5p binds to the 3'UTR of TRAF6 (upper panel, Figure 2B). In addition, analysis from a database (12) (GSE16969) indicates a relative strong negative relationship between the expression of miR-322-5p and the incidence of S.E. (miR-322-5p was found lower in the patients with intractable epilepsy, lower panel, Figure 2B). Our in vivo data supported this notion where the level of miR-322-5p sharply reduced upon the S.E. induction by pilocarpine (one week after induction) and continued to decrease over the next 3 weeks until reached the lowest level (approximately 50\% compared to that in the control counterparts) (Figure $2 \mathrm{C}$ ). Subsequently, our qPCR analyses of the S.E. rat brain tissues also demonstrated a significantly increased mRNA level of TRL4, TRAF6, IRF5, IL-1 $\beta$, and RelA (Figure 2D).

The LPS-induced inflammatory circuit in GABAergic M213-20 cells was negatively regulated by miR-322$5 p$ 
Next, we attempted to simulate the neuroinflammatory reactions by treating M213-20 cells with LPS. We found that LPS dose-dependently decreased the cell viability of M213-20 cells (Figure 3A). Next, western blots of LPS-treated M213-20 cells demonstrated dose-dependent decreased expression of inflammatory markers including TRAF6, TRL4, RelA, and IL-6 (Figure 3B). Consistently, the miR-322-5p level was significantly lower in LPS-treated M213-20 cells compared to their nontreated counterparts (Figure 3C). Subsequently, we manipulated the level of miR-322-5p using antagomir (downregulation) and mimic (upregulation) molecules in M213-20 cells. Antagomir transfection led to the significantly increased expression of TRAF6 (its target), IRF5, and RelA, while decreased expression of GABA and GAD1 (Figure 3D); this phenomenon was reversed in mimic-transfected M213-20 cells (Figure 3D).

\section{Exogenous miR-322-5p treatment significantly reduced the neuroinflammation}

After establishing that the reduced level of miR-322-5p in the S.E. rats was associated with the increased TRAF6/NF-kB inflammatory circuit, we aimed to determine the therapeutic potential of miR-322-5p. The brain tissues from rats that were injected with exogenous miR-322-5p immediately post S.E. induction clearly showed a higher number of viable neurons in the CA3 region compared to the S.E. rats without treatment (Figure 4A). In addition, rats injected with miR-322-5p showed a significantly lower level of neuroinflammation, as reflected by the reduced expression of TRAF6, RelA, IL-6, and TRL4 than the S.E. rats without treatment (Figure 4B). The level of miR-322-5p was observed to be elevated in the miR-322$5 p$ injected rat brain compared to their control counterparts (Figure 4C). Finally, the western blots of the brain homogenates demonstrated that a lower expression of neuroinflammatory markers including TRAF6, TLR4, RelA, and increased expression of GAD1 and GABA in the rat brain received

\section{Discussion}

Accumulating evidence indicates a close correlation between chronic inflammation and epileptogenesis in patients. However, the signaling network underlying responsible for the persistence of neuroinflammation remains to be explored. The classical TLR4/NF-kB signaling pathway is established to be responsible for inflammatory responses in different diseases including epilepsy (13). It has been postulated that prolonged use of anti-epileptic drugs may contribute towards acute/chronic inflammation, oxidative stress, leading to recurrent epilepsy, and resistance to drugs(14). In this study, we identified elevated TLR4/IRF5/NF-kB signaling in the brain of S.E. rats. Previous studies demonstrated that the inflammatory mediators, such as tumor necrosis factor (TNF)-a, IL-1 $\beta$, and IL- 6 may contribute towards epileptic development and progression. More importantly, elevated expression of TLR4 was identified in surgical resection specimens of drug-resistant temporal lobe epilepsy (TLE), focal cortical dysplasia (FCD), and tuberous sclerosis complex (TSC) patients (15). The inhibition of HMGB1/TLR4 was shown to reduce the incidence of seizures (16). Moreover, TRAF6, a downstream effector of TRL4 signaling, has been shown to play an essential role in Th1 type inflammation and involved in inhibiting autophagy and promoting oxidative stress in hemorrhaged rat brain (17). More importantly, the inhibition of TLR-4/NF-KB signaling and IL-1 $\beta$ in the hippocampus was shown to attenuate the severity of S.E. in 
rats(18). These findings signified that TLR4/NF-kB signaling could play an important role in the development of epilepsy.

Here, we demonstrated another potential regulatory mechanism by which TRL4 signaling was amplified in the S.E. rat brains. miR-322-5p was one of the most reduced microRNA species after the S.E. establishment in our study as well as in another study (11). Our results showed that miR-322-5p downregulated TRAF6 and IRF5, both activating components of the NF-kB signaling pathway. A previous study indirectly supported our notion where an increased level of miR-322-5p elevated EZH2 and activated Akt/GSK3 $\beta$ pathway, thereby protecting myocardial cells from ischemic reperfusion injury (19). On the contrary, in the S.E. rat brain, miR-322-5p was significantly reduced and associated with a higher level of neruoinflammation.

We then verified these observations in GABAergic M213-20 cells where LPS treatment significantly induced the TLR4/IRF5/NF-kB inflammatory circuit. Previous reports also supported that peripheral inflammation contributed to the development of seizures and TLR4 signaling appeared to be instrumental (20). Inflammatory cytokines such as IL-6 and IL-1 $\beta$ significantly increased upon TLR4 activation and consistently reported in epileptic patients, indicating a chronic inflammatory state (21). In addition, increased IL- 6 was shown to mediate the degradation of GABAergic neurons in the mouse brain (22). Our findings were in agreement with these reports where increased IL- 6 and IL-1ßlevel was associated with decreased GABA and GAD1 expression. More importantly, we provided evidence that exogenous miR-322-5p (using mimic molecules) resulted in the restoration of GAD1 and GABA in LPStreated M213-20 cells, accompanied with a signficiantly reduced expression of the neuroinflammatory markers, NF-kB, TRL4 and IRF.

Finally, we examined the feasibility of exogenous miR-322-5p as a Preventive measure. Rats injected with exogenous miR-322-5p immediate after S.E. induction showed a significantly lower expression of TLR4, IRF5, and NF-kB, while increasing GAD1 and GABA, compared to their counterparts which received scramble. This finding suggested the feasibility of exogenous miR-322-5p as an inhibitor of neuroinflammation. Emerging evidence demonstrates that stereotactic injection or antagomirs or mimic molecules into the hippocampal regions not only is feasible but also reduces the incidence of seizures in rodent models (23). Thus, our findings provided support for injecting exogenous miR-322-5p as a Preventive measure for reducing chronic neuroinflammatory circuits, namely, the TRL4/TRAF6/NF-kB pathway.

\section{Conclusions}

In the graphical summary of Figure 5, the present study provided preclinical in vitro and in vivo models demonstrating the essential role of TRL4/TRAF6/NF-kB as a neuroinflammatory signaling pathway associated with pilocarpine-induced epilepsy; miR-322-5p functioned as a negative regulator of this inflammatory circuit and could serve as a potential therapeutic tool for suppressing chronic inflammation 
and preventing the decreased level of GAD1 and GABA, thereby reducing the incidence of epileptic episodes. Further investigation is warranted for the safety of exogenous miR injection.

\section{Abbreviations}

miRNAs : MicroRNAs ; SE: status epilepticus; GABA : Aminobutyric acid ; GAD1: glutamate decarboxylase 1; CNS : central nervous system; TLRs : Toll-like receptors; HMGB1: high-mobility group box-1; 3'UTR : $3^{\prime}$ untranslated region; LiCl: lithium chloride

\section{Declarations}

\section{Acknowledgments}

This work was supported by the Science and Technology Program of Zhejiang province (No. 2018C37086), the Traditional Chinese Medicine Science and Technology Program of Zhejiang province (No. 2020ZB022).

\section{Authors' contributions}

QZ and WW designed the research subject. BH and WY conducted literature retrieval and screening, and $\mathrm{BH}$ and HK conducted laboratory testing. QZ wrote the manuscript. WW critically revised the manuscript. All authors read and approved the final manuscript.

\section{Availability of data and materials}

All data generated or analyzed during this study are included in this published article.

\section{Ethics approval and consent to participate}

All procedures were conducted according to the regulations of Animal experiment facility and approved by the Experimental Animal Ethical Committee of Zhejiang Provincial People's Hospital.

\section{Consent for publication}

Not applicable.

\section{Competing interests}

The authors declare that they have no competing interests.

\section{Conflict of interest statement}

The authors declare that there are no potential conflicts of interest.

\section{References}


1. Betjemann JP, Josephson SA, Lowenstein DH, Burke JF. Trends in Status Epilepticus-Related Hospitalizations and Mortality: Redefined in US Practice Over Time. JAMA Neurol. 2015;72(6):650-5.

2. Vezzani A, Aronica E, Mazarati A, Pittman QJ. Epilepsy and brain inflammation. Experimental neurology. 2013;244:11-21.

3. Spatola M, Dalmau J. Seizures and risk of epilepsy in autoimmune and other inflammatory encephalitis. Current opinion in neurology. 2017;30(3):345-53.

4. Suleiman J, Dale RC. The recognition and treatment of autoimmune epilepsy in children. Developmental medicine and child neurology. 2015;57(5):431-40.

5. Chen L, Zheng H, Zhang S. Involvement of upregulation of miR-210 in a rat epilepsy model. Neuropsychiatric disease and treatment. 2016;12:1731-7.

6. Hu K, Xie YY, Zhang C, Ouyang DS, Long HY, Sun DN, et al. MicroRNA expression profile of the hippocampus in a rat model of temporal lobe epilepsy and miR-34a-targeted neuroprotection against hippocampal neurone cell apoptosis post-status epilepticus. BMC neuroscience. 2012;13:115.

7. García-García L, Shiha AA, Fernández de la Rosa R, Delgado M, Silván Á, Bascuñana P, et al. Metyrapone prevents brain damage induced by status epilepticus in the rat lithium-pilocarpine model. Neuropharmacology. 2017;123:261-73.

8. Reddy DS, Kuruba R. Experimental models of status epilepticus and neuronal injury for evaluation of therapeutic interventions. International journal of molecular sciences. 2013;14(9):18284-318.

9. Gao F, Gao Y, Liu YF, Wang L, Li YJ. Berberine exerts an anticonvulsant effect and ameliorates memory impairment and oxidative stress in a pilocarpine-induced epilepsy model in the rat. Neuropsychiatric disease and treatment. 2014;10:2139-45.

10. Korzhevskii DE, Otellin VA. Immunocytochemical detection of astrocytes in brain slices in combination with Nissl staining. Neuroscience and behavioral physiology. 2005;35(6):639-41.

11. Bot AM, Dębski KJ, Lukasiuk K. Alterations in miRNA levels in the dentate gyrus in epileptic rats. PloS one. 2013;8(10):e76051.

12. Boer K, Crino PB, Gorter JA, Nellist M, Jansen FE, Spliet WG, et al. Gene expression analysis of tuberous sclerosis complex cortical tubers reveals increased expression of adhesion and inflammatory factors. Brain Pathol. 2010;20(4):704-19.

13. Devinsky O, Vezzani A, Najjar S, De Lanerolle NC, Rogawski MA. Glia and epilepsy: excitability and inflammation. Trends in neurosciences. 2013;36(3):174-84.

14. Kalilani L, Sun X, Pelgrims B, Noack-Rink M, Villanueva V. The epidemiology of drug-resistant epilepsy: A systematic review and meta-analysis. Epilepsia. 2018;59(12):2179-93.

15. Kleen JK, Holmes GL. Taming TLR4 may ease seizures. Nat Med. 2010;16(4):369-70.

16. Zhao J, Wang Y, Xu C, Liu K, Wang Y, Chen L, et al. Therapeutic potential of an anti-high mobility group box-1 monoclonal antibody in epilepsy. Brain Behav Immun. 2017;64:308-19.

17. Dou Y, Shen H, Feng D, Li H, Tian X, Zhang J, et al. Tumor necrosis factor receptor-associated factor 6 participates in early brain injury after subarachnoid hemorrhage in rats through inhibiting autophagy 
and promoting oxidative stress. Journal of neurochemistry. 2017;142(3):478-92.

18. Qu Z, Jia L, Xie T, Zhen J, Si P, Cui Z, et al. (-)-Epigallocatechin-3-Gallate Protects Against LithiumPilocarpine-Induced Epilepsy by Inhibiting the Toll-Like Receptor 4 (TLR4)/Nuclear Factor-kB (NF-kB) Signaling Pathway. Medical science monitor : international medical journal of experimental and clinical research. 2019;25:1749-58.

19. Dong W, Xie F, Chen XY, Huang WL, Zhang YZ, Luo WB, et al. Inhibition of Smurf2 translation by miR$322 / 503$ protects from ischemia-reperfusion injury by modulating EZH2/Akt/GSK3 $\beta$ signaling. American journal of physiology Cell physiology. 2019;317(2):C253-c61.

20. Vezzani A, Moneta D, Richichi C, Perego C, De Simoni MG. Functional role of proinflammatory and anti-inflammatory cytokines in seizures. Advances in experimental medicine and biology. 2004;548:123-33.

21. Uludag IF, Duksal T, Tiftikcioglu BI, Zorlu Y, Ozkaya F, Kirkali G. IL-1 $\beta$, IL-6 and IL1Ra levels in temporal lobe epilepsy. Seizure. 2015;26:22-5.

22. Dugan LL, Ali SS, Shekhtman G, Roberts AJ, Lucero J, Quick KL, et al. IL-6 mediated degeneration of forebrain GABAergic interneurons and cognitive impairment in aged mice through activation of neuronal NADPH oxidase. PloS one. 2009;4(5):e5518.

23. Huang H, Cui G, Tang H, Kong L, Wang X, Cui C, et al. Silencing of microRNA-146a alleviates the neural damage in temporal lobe epilepsy by down-regulating Notch-1. Molecular brain. 2019;12(1):102.

\section{Table}

Table 1. Primer sequnces for real-time PCR reactions

\begin{tabular}{|lll|}
\hline Gene name & Forward & Reverse \\
miR-322-5p & AGCGTGCTGTGCGTGTGAC & CAGTGCAGGGTCCGAGGTATT \\
\hline GABA & AATGGGCGGATTGGTGTC & TCATCTTGGGAGGGCTGT \\
\hline GAD1 & TCTTTTCTCCTGGTGGTGCC & TCATCTTGGGAGGGCTGT \\
\hline IL-6 & TCCTACCCCAACTTCCAATGCTC & TTGGATGGTCTTGGTCCTTAGCC \\
\hline IL-1b & CACCTCTCAAGCAGAGCACAG & GGGTTCCATGGTGAAGTCAAC \\
\hline NF-kB (RelA) & AATTGCCCCGGCAT & TCCCGTAACCGCGTA \\
\hline TRAF6 & CAGTCCCCTGCACATT & GAGGAGGCATCGCAT \\
\hline IRF5 & ATGCTGCCTCTGACCGA & GCCGAAGAGTTCCACCTG \\
\hline GAPDH & AGCCACATCGCTCAGACA & TGGACTCCACGACGTACT \\
\hline
\end{tabular}

\section{Figures}


A

Control

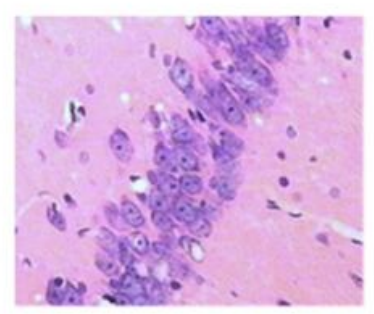

SE

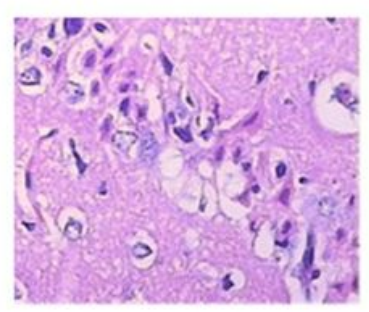

B

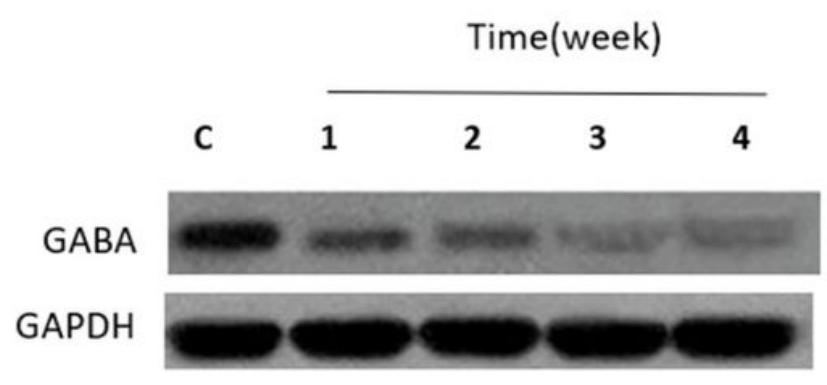

C

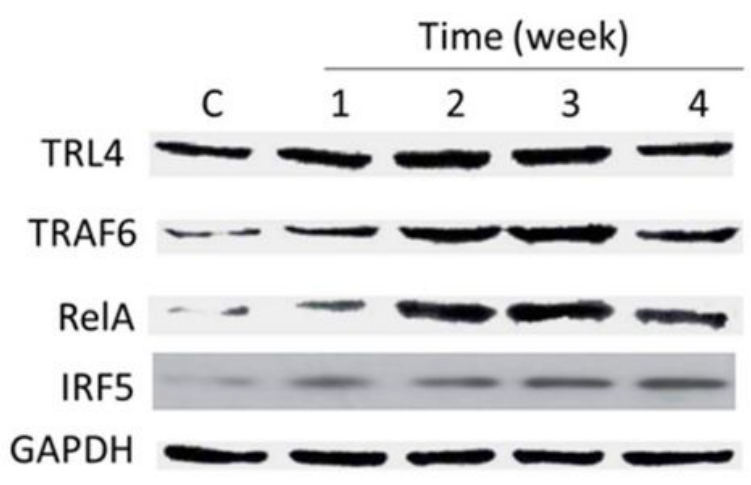

Figure 1

Increased neuroinflammatory circuit, TLR4/TRAF6/NF-kB in status epilepticus (S.E.) rats. (A) Representative brain sections demonstrated the dis-organization of neurons within the CA3 region of S.E. rat brain compared to the normal counterparts. (B) Decreased GABA secretion in the S.E. rat brains. (C) Western blot of rat brain revealed that the increased expression of TRAF6, TRL4, IRF5, and RelA post S.E. induction. 
B

\begin{tabular}{|l|l|l|}
\hline miRNA_ID & P.Value & logFC \\
\hline rno-miR-322-5p & 0.000496 & -0.1688 \\
\hline rno-miR-16-3p & 0.001259 & 0.1356 \\
\hline rno-miR-592 & 0.001507 & -0.1746 \\
\hline rno-miR-493-3p & 0.003205 & 0.1422 \\
\hline rno-miR-218a-1-3p & 0.004292 & -0.1154 \\
\hline rno-miR-7b & 0.004863 & -0.3904 \\
\hline rno-miR-1224 & 0.005434 & -0.2308 \\
\hline rno-miR-26b-3p & 0.005473 & -0.314 \\
\hline rno-miR-34c-3p & 0.005515 & 0.2422 \\
\hline rno-miR-96-5p & 0.005533 & -0.0892 \\
\hline
\end{tabular}

TRAF6 5' gugGAGAGGUGACUGUCGUGCUGCUg 3' $:|:|:||||||||||||$
miR-322-5p 3' aggUUUUGUACUUA----ACGACGAC 5'
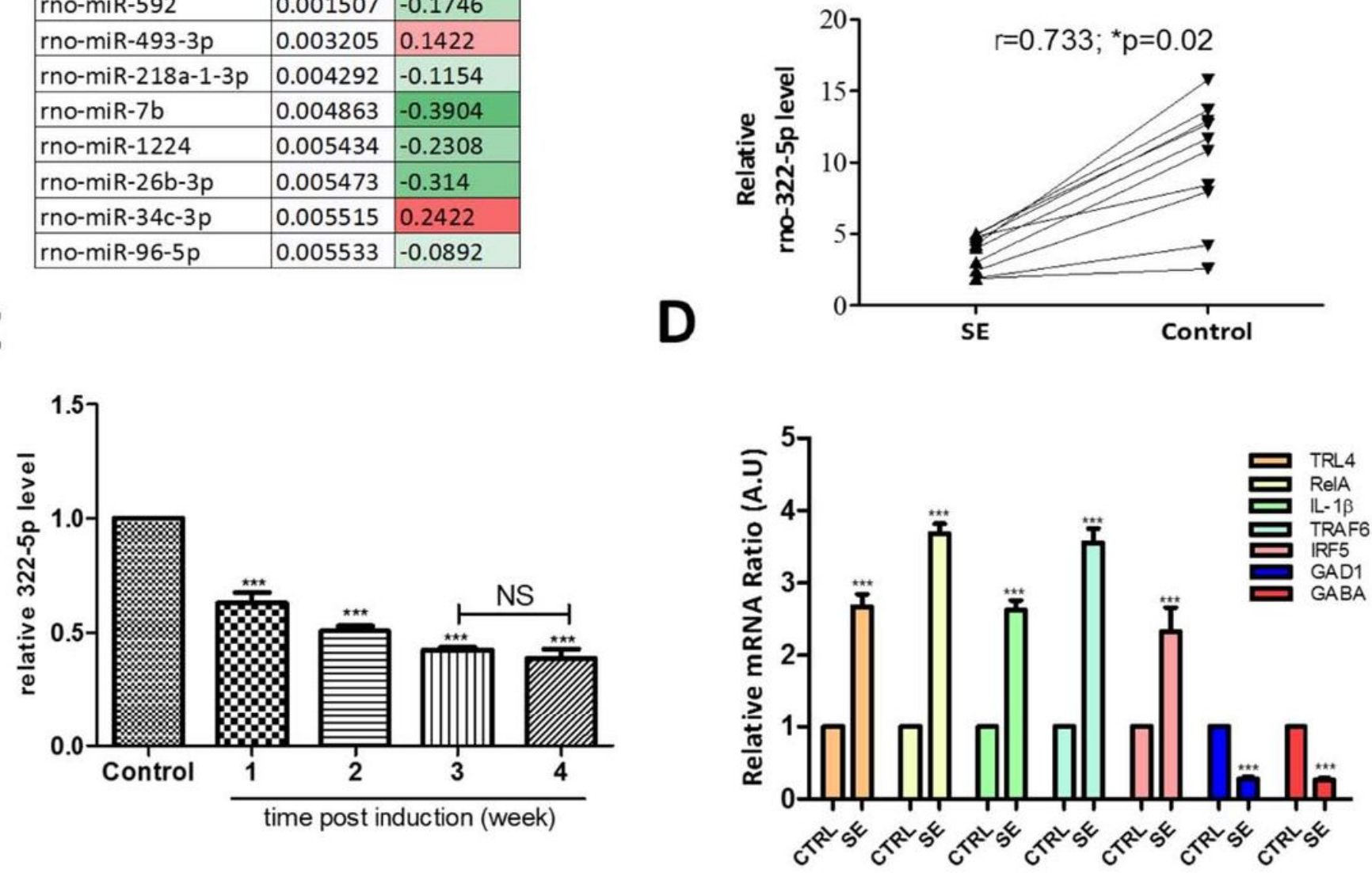

Figure 2

A decreased miR-322-5p was associated with the increased neuroinflammatory circuit. (A) miR-322-5p was identified as one of the most decreased microRNA species post S.E. induction in rats' brain (GSE49850). (B) Insert demonstrates the binding sequences of miR-322-5p and the 3'UTR of TRAF6. Lower the panel, a relatively strong negative correlation was identified between the expression of miR322-5p and TRAF6 mRNA in patients with intractable epileps. (C) Real-time PCR analysis of mRNA isolated from S.E. rat brains indicated a significantly increased miR-322-5p in the S.E. rats post induction. (D) Comparative qPCR analysis between control and S.E. rat brains. Neuroinflammatory markers including TRL4, IRF5, RelA (NF-kB subunit), and IL-1 $\beta$ were significantly increased while GAD1 and GABA decreased in the S.E. samples. ${ }^{*} \mathrm{P}<0.05 ;{ }^{* *} \mathrm{P}<0.01 ; * \star * \mathrm{P}<0.001$. 


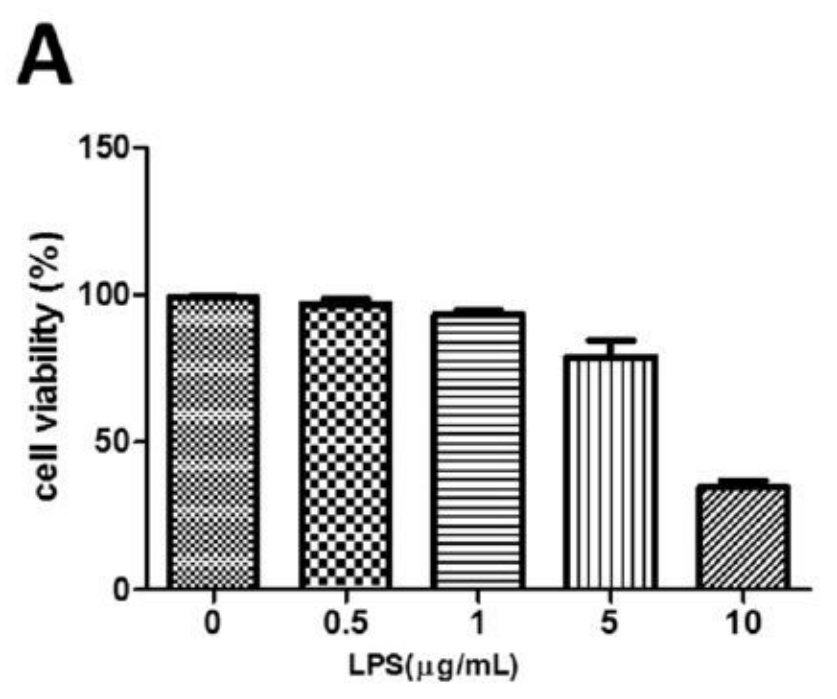

B
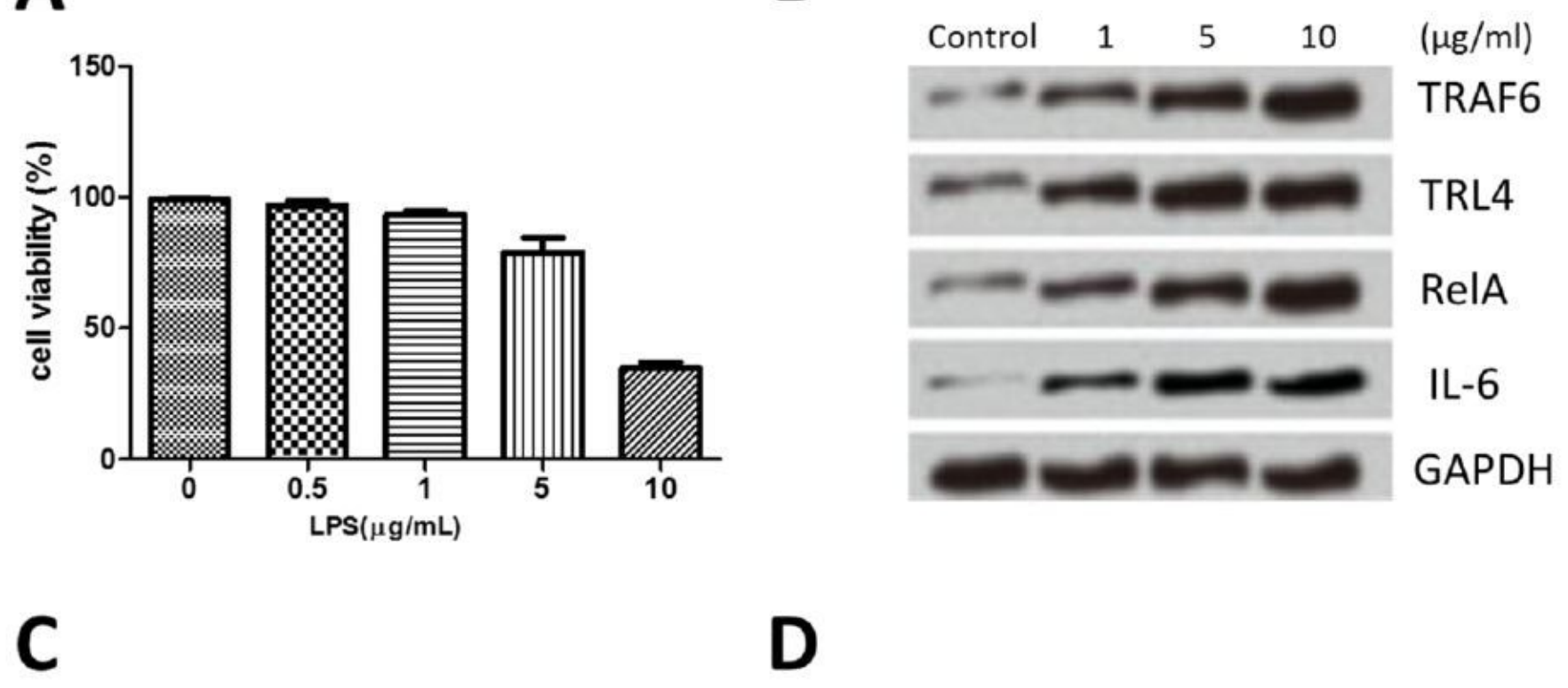

D
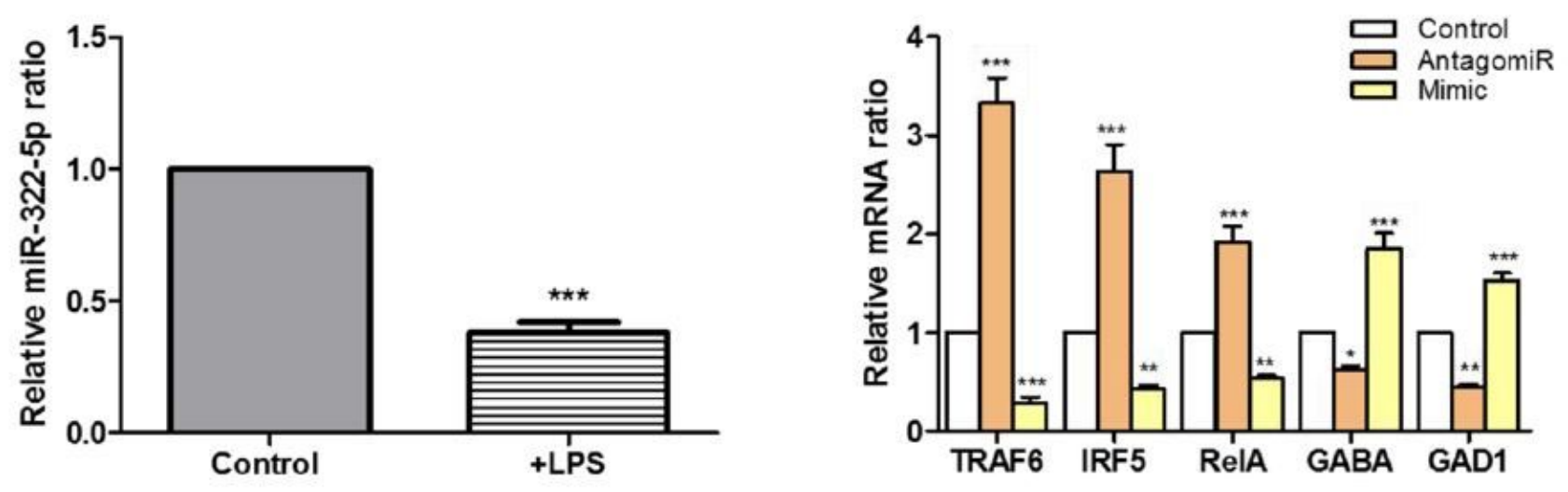

Figure 3

LPS-induced apoptosis and inflammation in GABAergic M213-20 cells were associated with the downregulation of miR-322-5p. (A) GABAergic M213-20 cells were treated with LPS under a range of concentrations $(0.5,1,5$, and $10 \mu \mathrm{g} / \mathrm{ml})$ for $12 \mathrm{~h}$. Cell viability was measured by CCK-8 assay kit. (B) Western blot showed an increase in the expression of inflammation-associated markers. (C) Real-time PCR analysis. The miR-322-5p level was significantly lower in the LPS-treated M213-20 cells compared to the nontreated counterpart. (D) Comparative GPCR analyses of the neuroinflammatory circuit in association with miR-322-5p levels. Antagomir (downregulation) transfected cells led to significantly increased inflammatory markers, TRAF6, IRF5, and RelA, while reducing GABA and GAD1. * $P<0.05$, * $P$ $<0.01, \star \star \star ~ P<0.001$. 
A

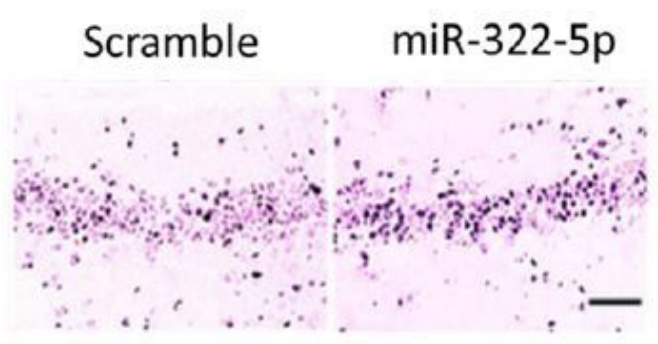

C

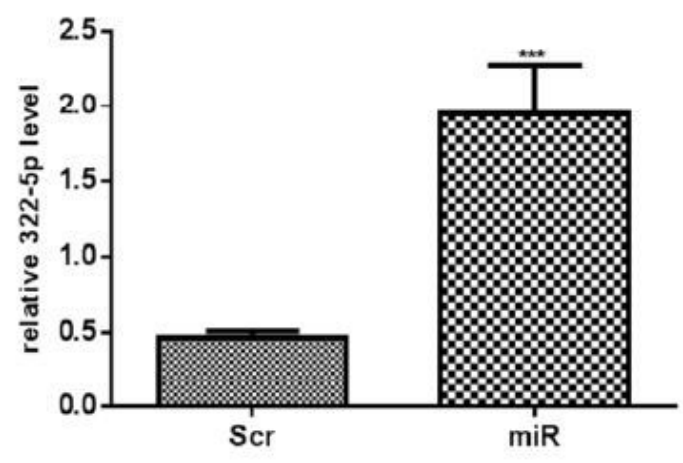

B

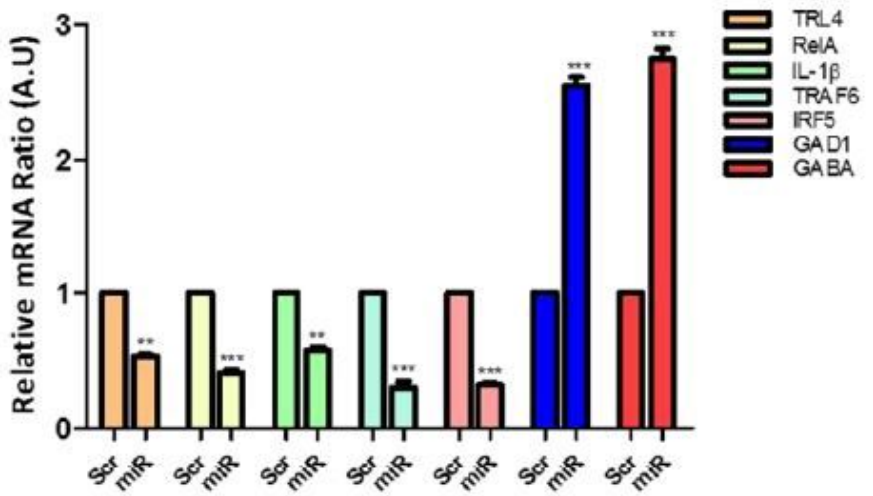

D

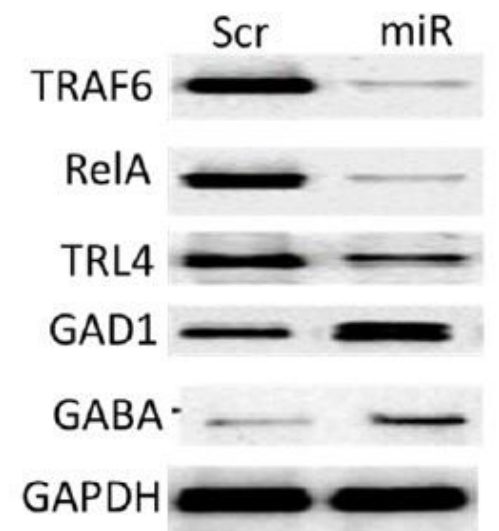

Figure 4

(A) Representative micrographs of the Nissl-stained CA3 region of rat hippocampus from both scramble and miR-322-5p injected S.E. rats. Neurons with intact morphology were identified as surviving neurons. Scale bars: $20 \mu \mathrm{m}$. (B) Comparative qPCR analysis of the brain tissues from scramble and miR-322-5p injected rats. A significantly lower levels of TRL4, TRAF6, IRF5, IL-6, and NF-kB were observed in the miR322-5p group as compared with the scramble group. (C) Similarly, the miR-322-5p injected rat brain contained significantly higher miR-322-5p than the ones from the control group. (D) Western blot of the brain homogenate. The neuroinflammatory markers, TLR4, RelA (NF-kB), and TRAF6 were decreased compared to the scrambling control tissues. 
Pilocarpine-induced epilepsy

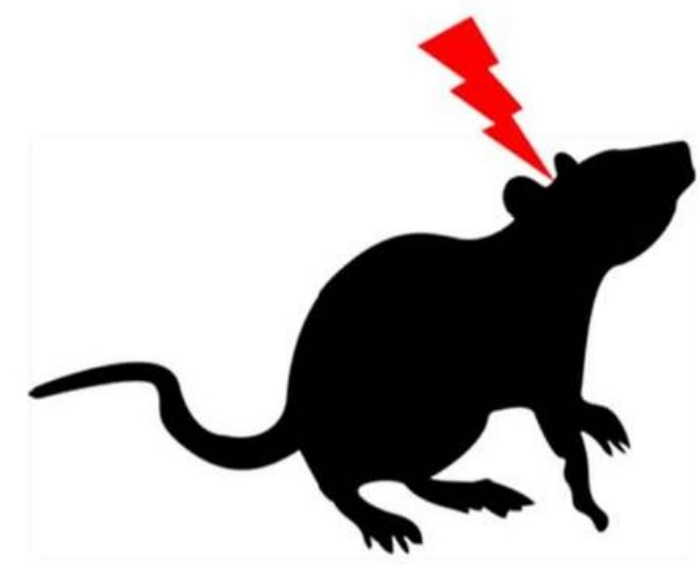

TLR4

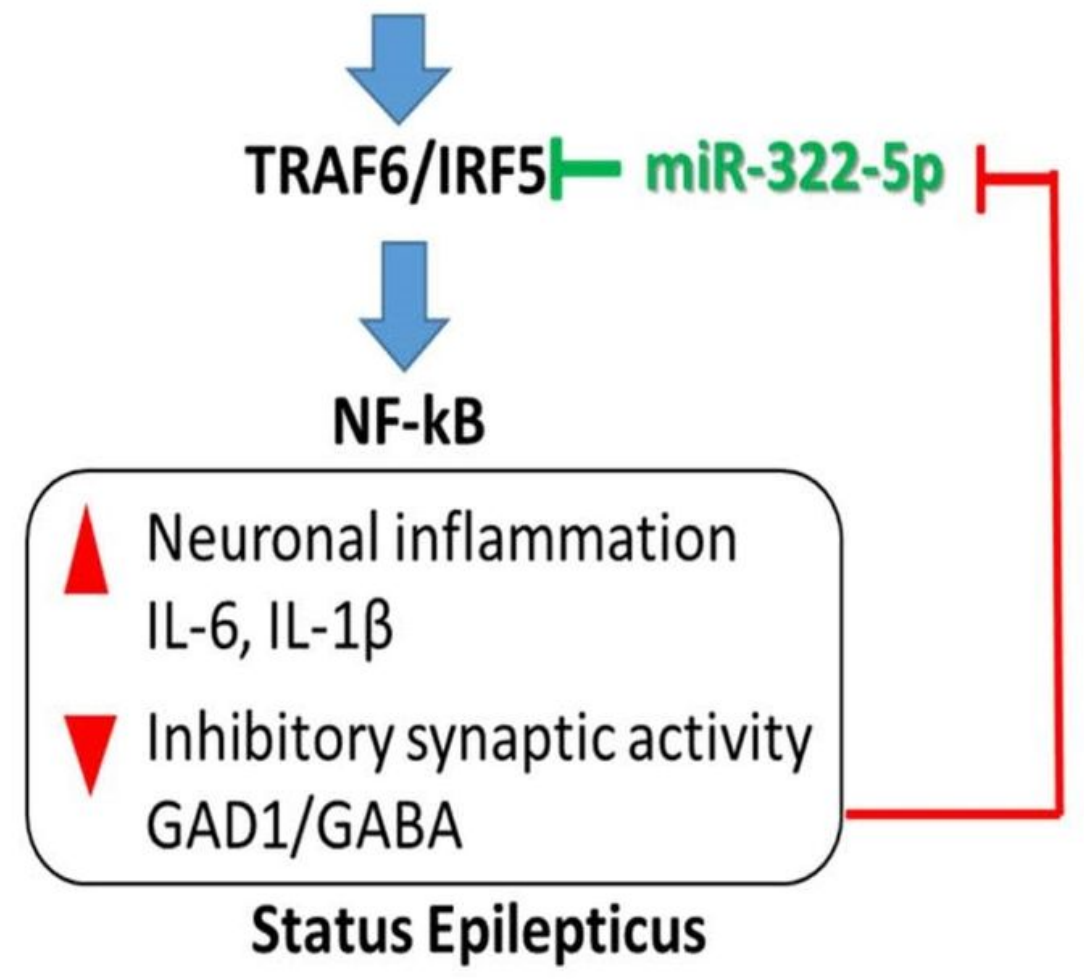

Figure 5

A pilocarpine-induced rat epileptic model was created to demonstrate the important role of the neuroinflammatory circuit, TRL4/TRAF6/NF-kB in the epileptogenic process. In the status epilepticus (S.E) rat brain, miR-322-5p was found significantly lower than the normal counterparts and TRAF6 and IRF5 were found to be miR-322-5p's targets. An increased level of miR-322-5p was shown to significantly reduce the neuroinflammatory markers and restore the level of GAD1 and GABA. 